\title{
Alcohol Exposure and Cognitive Development: An Example of Why We Need a Contextualized, Dynamic Life Course Approach to Cognitive Ageing - A Mini-Review
}

\author{
Kaarin J. Anstey \\ Ageing Research Unit, Centre for Mental Health Research, The Australian National University, \\ Canberra, A.C.T., Australia
}

\section{Key Words}

Lifespan psychology • Alcohol • Cognition • Dementia •

Life course epidemiology

\begin{abstract}
Background: A substantial literature exists that demonstrates associations between putative risk factors and cognitive decline in late life. However, there is a need to integrate this broad literature within a framework that incorporates the interaction of behavioral and ecological influences with cognitive development. Such a framework is required for developing a range of personal and environmental interventions to optimize cognitive development in the population, and to reduce the risk of cognitive impairment in late life. Objective: This review aims to identify the key considerations for developing a life course model of the various factors that influence cognitive development and cognitive decline. A contextualized, dynamic approach to life course epidemiology is proposed. Methods: A theoretical evaluation of key methodological and interpretational issues relating to how risk factors influence cognitive development and cognitive impairment was conducted. This focused on the example of alcohol consumption as a risk factor for cognitive decline and dementia. Results: This review identified dimensions that need to be accounted for in life course theories of cognitive development and cognitive impairment.
\end{abstract}

These include: (a) intergenerational influences; (b) methodological and interpretational issues; (c) individual differences (personal factors); (d) contextual factors (environmental or ecological factors), and (e) cognitive ability as determinant. The methodological and interpretational factors included measurement of exposure and outcome variables; the important distinction between level of ability versus change over time; nonlinear relationships among exposures and outcomes, and outcomes and age; the distinction between association and cause, and between short-term effects and long-term change. Conclusion: A contextualized, dynamic approach to life course epidemiology accounts for the complex range of influences over the life course that interact to determine normal and pathological cognitive ageing. This approach provides a framework for the development of interventions to maximize cognitive gains in early life, and minimize cognitive loss in late life.

Copyright $\odot 2008$ S. Karger AG, Basel

\section{Life Course Epidemiology and Lifespan Psychology}

Several authors have provided excellent descriptions of life course epidemiology [1-5] that provide a framework for studying cognitive and chronic disease outcomes in later life. This general framework argues that individuals' specific health outcomes are the result of a

\section{KARGER}

Fax +41613061234 E-Mail karger@karger.ch www.karger.com
(C) 2008 S. Karger AG, Basel

0304-324X/08/0545-0283\$24.50/0

Accessible online at:

www.karger.com/ger
Kaarin J. Anstey

Ageing Research Unit, Centre for Mental Health Research

The Australian National University

Canberra, ACT 0200 (Australia)

Tel. +61 2612 58410, Fax +61 2612 50733, E-Mail kaarin.anstey@anu.edu.au 
complex system of exposures that interact with biological processes over the lifespan to cause disease. Key concepts are that disease outcomes are associated with exposures early in life or at specific periods during the lifespan. Risk factors are thought to have either independent or cumulative effects, either clustering at particular stages of the life course or creating a chain of risks whereby one risk factor leads to another $[5,6]$. Life course approaches also include the concept of critical periods, where risk factors or exposures at specific development stages alter the underlying structure of the organism, altering its innate biological 'programming'. These approaches also include 'effect modifiers' which are variables that modify the level of the effect of an exposure occurring earlier in the life course [6].

Lifespan approaches have been used in developmental psychology to explain ageing since the 1960s [7]. Early lifespan models of cognitive development proposed by Horn [8], and Baltes and Reinert [9] and Baltes et al. [10, 11], incorporated many of the factors described in life course theories of dementia $[12,13]$, but focused on normative development rather than disease. For example, Horn [14] described the determinants of fluid and crystallized intelligence, including genetics, acculturation, environment, injury and accident, and discovered that specific cognitive abilities are affected by different exposures over the lifespan. The notion of critical period is also a central concept to lifespan theories of animal behavior and learning, as substantial behavioral and physiological evidence shows that exposure during critical periods of development permanently shape brain development. In some cases, deprivation of specific stimuli leads to irreversible lack of development of adaptive functions [15].

An important, but not exclusive distinction between psychologically oriented approaches and lifespan epidemiological approaches is that psychological theories primarily focus on normative human development, whereas epidemiological approaches primarily focus on disease outcomes. Integrating normative theories of cognitive ageing with life course epidemiological approaches allows for a more comprehensive formulation of the complex and varied influences that affect cognitive development and the development of cognitive impairment in ageing. For example, appreciation of the differential rates of growth and decline in fluid and crystallized abilities informs the clinical classification of cognitive impairment. The clinical assessment of premorbid intellectual function draws on the knowledge of crystallized abilities that are less susceptible to neurological damage.

\section{A Contextualized, Dynamic Approach}

To fully capture the dynamic nature of cognitive development over the life course, cognitive function and human behavior must be incorporated into models as causal agents [16]. People interact with their environment to shape the way environments in turn influence cognitive development. Such interactions may occur at the individual level (e.g. personally deciding to increase physical activity) or societal level (e.g. providing more opportunities and reward for engaging in physical activity, reducing overall lead exposure in mining communities).

Improving cognitive development through education and lifestyle interventions requires manipulation of both the environment (e.g. schooling and learning environments, reducing exposure to neurotoxins) and human behavior (e.g. avoidance of toxins, engagement in physical and mental activity). Therefore, the person is not a passive recipient of the effects of risk factors on cognitive and brain development, but actively interacts with the environment through their behavior which is in turn influenced by their knowledge of, and motivation to engage in, health-promoting behaviors. The development and implementation of interventions to optimize cognition requires identification of the many environmental and behavioral factors that influence cognitive development. It also requires identification of the contextual, cognitive and behavioral factors that influence the uptake and maintenance of individual and social strategies that lead to optimal cognition.

\section{The Need for Specific Content Knowledge within}

General Frameworks

Within a general lifespan approach that incorporates social and historical context, as well as human agency, specific methodological and content knowledge is required to evaluate each potential influence on cognitive development. Beyond general principles and paradigms, this includes the awareness of issues relating to measurement and longitudinal methodologies, and knowledge of culture, diet, biology, gene-environment interactions, brain development and the influence of other contextual and intrapersonal variables. An understanding of how different predictors or exposures interact with each other is sometimes necessary to fully explain influences on cognitive development. The study of risk factors and exposures has its roots in epidemiology, while the knowledge based on cognitive development and theories of contextual effects draws from psychology [17]. Hence, the work described here draws from both life course epidemiology and lifespan psychology. Five specific consider- 
ations for a contextualized, dynamic lifespan approach are identified, using alcohol consumption as an example exposure variable.

\section{Intergenerational Influences on Cognitive Development}

In addition to genetic characteristics, social and biological contexts may be transmitted from one generation to its offspring. This in turn influences health outcomes and cognitive development [18]. For example, there is substantial evidence to show that alcohol exposure in utero may affect later cognitive development. The most extreme consequence is fetal alcohol syndrome (FAS) which involves growth retardation and facial changes due to the effects of alcohol on tissue development of the fetus during weeks 4-8. FAS also affects normal central nervous system development with reductions in brain volume or subvolumes and changes to nerve cells and synapses [19]. Cognitive deficits associated with FAS include impairment in overall intellectual functioning, motor skills and learning [20]. Although FAS results from high levels of alcohol exposure, lower levels of exposure have also been associated with deficits in fetal development that do not reach diagnostic criteria for FAS. It remains unclear how reversible the central nervous system changes caused by alcohol exposure are, and which level of exposure is considered dangerous for normal fetal development. Other factors, such as gender, timing of exposure, genetic differences, and placental sufficiency, may function to moderate the extent to which a mother's alcohol consumption affects her fetus [21].

The consideration of intergenerational influences on cognitive development is analogous to Barker's fetal origin's hypothesis [22] which was initially proposed to explain how a poor fetal environment increased the risk of cardiovascular disease in later life. Others have extended the paradigm of linking the quality of prenatal experience to adult risk for disease to the areas of cognition. Vulnerabilities may be established or 'programmed' early in life, increasing the individual's susceptibility to dementia in late life [23].

\section{Methodological and Interpretational Issues}

Interpretation of research findings requires awareness of methodological and data analytic issues. Observations taken from different phases during a life course can rare- ly be based only on one study, due to the long time periods involved. This increases the importance of issues associated with measurement and interpretation. Several of the key methodological and interpretational issues are described below.

\section{Measurement of Exposures and Outcomes}

Accurate measurement of exposure variables and cognitive outcomes is essential for evaluating the effect of exposures on cognitive development. It is often difficult to obtain sensitive measures of risk factors, either because they may have occurred prior to the measurement occasion or are difficult to measure. In the case of alcohol exposure, most information is obtained by self report, and individuals are often not aware of the percentage of alcohol contained in specific drinks or the volume of drinks consumed [24]. This differs from the measurement of clinical risk factors for cognitive decline, such as hypertension or serum cholesterol. Moreover, establishing the effect of alcohol on cognitive development may require obtaining information on past drinking. The reliability of such data [25] varies according to the wording of questions and whether trained interviewers complete questionnaires on behalf of participants or whether participants complete questionnaires confidentially. Countries use different definitions of a 'standard drink' [26], hence international comparisons need to be conducted by converting measures to a single metric (e.g. grams per liter). The evaluation of the effects of alcohol consumption on cognition is further complicated by differences in type of beverage. Some research has suggested that red wine has specific benefits for cognition compared with beer, while other studies have not found a beverage-specific effect [27]. For example, a 2-year follow-up of 2,632 participants [28] found that light to moderate beer drinkers had a higher risk of dementia, but that light to moderate wine drinkers had a lower risk of dementia, compared with nondrinkers [29].

Without adequate methods for measuring exposure it is not possible to establish its effects, and this may lead to a lack of scientific data on potential neurotoxins in the general community. For example, there is circumstantial evidence showing that heavy exposure to insecticides is associated with cognitive deficits, but few studies have developed measures sensitive enough to determine whether everyday insecticide exposure in the community is a risk factor for cognitive decline [29].

One of the main difficulties for studying cognitive development over the lifespan is that as abilities change from childhood to adulthood, different tests may be re- 
quired to prevent ceiling or floor effects, and to ensure construct validity. Most longitudinal studies of cognitive development therefore need to manage the issue of measurement invariance in their outcomes. This differs from research where the outcome is a disease state (e.g. cancer vs. no cancer).

\section{Level of Ability versus Rate of Change in Ability over Time}

When describing longitudinal findings, a fundamental principle is the difference between the association a risk factor has with level of performance, versus change in performance. As has been widely argued [30], it is not necessarily the case that a factor that is associated with between-person differences in cognitive ability (level) will also influence cognitive change.

This issue is highlighted by the difficulty in interpreting associations based purely on data obtained in late life, and can be illustrated with the example of the association between cognitive performance and weekly alcohol consumption. A number of recent articles have shown that older adults who drink alcohol at light to moderate levels have better cognitive performance [31], reduced rates of cognitive decline in late adulthood and a reduced risk of dementia [32]. Drawing from research on alcohol and cardiovascular disease [33], several authors have concluded that for older adults, alcohol consumption in low to moderate doses may in fact be beneficial for the brain and cognition.

However, research conducted on younger adults has also found that light to moderate drinkers have better cognitive function than abstainers [34]. The interpretation of this result in relation to young adults has received a different emphasis. The focus here has been on why abstainers perform worse on cognitive tests than drinkers, because in young adults it is extremely unlikely that participants have been exposed to alcohol long enough for long-term effects to be evident. Moreover, cognitive abilities are still developing in young adults. When viewed from a lifespan perspective, the results suggest that alcohol abstainers, at all ages, have lower cognitive test scores. This means that it is possible that alcohol itself may have no effect on cognition, and that all we are seeing in late life is the selection effects that were already evident in early adulthood. Longitudinal studies that follow a sample of abstainers and drinkers from early adulthood are required to determine whether lifelong abstainers do have a different cognitive trajectory to that of light to moderate drinkers. Alternatively, randomized controlled trials over longer periods of time would be one method of determining whether alcohol does benefit cognition, but these raise ethical and practical issues and are hence not viable. Functional neuroimaging studies may also shed light on how alcohol affects brain function in the short term, and how brain function differs between harmful, moderate and nondrinkers. Longitudinal structural MRI studies will also elucidate whether differences derived from alcohol consumption between abstainers and moderate drinkers are due to selection effects or benefits.

\section{Nonlinear Relationships}

Another factor to consider is the possible nonlinear relationship between an individual risk factor and an outcome. Nonlinearity is also present in the relationship between cognitive development and age, and between the salience of risk factors and age. There may be optimal levels of exposures, so that little or too much may be detrimental for development.

A range of findings from the literature suggests that an inverse U- or J-shaped association exists between alcohol consumption and dementia risk, stroke, cognitive performance and cognitive decline [34-35]. This is supported by an inverse U-shaped association between markers of brain pathology and level of alcohol consumption in cross-sectional studies. For example, the Cardiovascular Health Study involving brain MRI scans of 3,660 adults aged 65 years or more found an inverse U-shaped association between white matter hyperintensities (WMHs) and alcohol consumption, with moderate alcohol consumption being associated with reduced prevalence of WMHs [35]. This study also found moderate drinking to be associated with reduced risk of cerebral infarction [36]. Likewise, the Rotterdam study also found an inverse U-shaped association between WMHs and alcohol consumption [37]. In contrast, the Atherosclerosis Risk in Communities Study of 1,909 adults aged 45-64 years found no association between alcohol consumption and either stroke risk or white matter grade [38]. However, these findings may be explained by limitations of the study design which did not include heavy drinkers and was based on a relatively young sample.

Although public health concern has often focused on problem drinking, findings from several studies show that light to moderate alcohol consumption is often associated with better outcomes than complete abstinence from drinking. Several studies have now reported that alcohol abstainers have lower scores on cognitive tests compared with moderate drinkers [31,39-42]. This effect has occurred on measures of a range of cognitive abilities including processing speed, memory, verbal ability, 
visuospatial ability and reasoning, in samples of young [34, 43], middle [39] and older adults [42], and across gender.

A 7-year longitudinal study of 1,277 adults aged 65 and older found that compared to abstention, light to moderate drinking was associated with less cognitive decline [44]. An 8-year follow-up of Japanese American community-dwelling elders also found that high levels $(\geq 26 \mathrm{~g}$ per day) of alcohol consumption were associated with greater cognitive decline than lower levels of alcohol consumption ( $<26 \mathrm{~g}$ per day) [45]. These results suggest that the inverse $U$ association holds for the relationship between cognitive decline and alcohol consumption. The inverse $\mathrm{U}$ association has also been found between alcohol consumption and stroke [46], but the pattern differs in that moderate drinkers appear not to be protected. The Cardiovascular Health Study followed 4,410 adults aged 65 and older for an average period of 9.2 years and found that adults consuming between 1 and 6 drinks per week (light drinkers) had a 20\% reduced risk of ischemic stroke compared with abstainers and those consuming more than 6 drinks per week. This is interesting because stroke is a risk factor for dementia and cognitive decline, illustrating a chain of risk associated with alcohol consumption and the nonlinear relationship between alcohol and stroke risk.

\section{Association versus Cause}

Although cross-sectional studies usually clearly state that causality cannot be assumed from their findings, results from longitudinal studies do not necessarily address the problem of causality. They may be more problematic because they are more likely to be cited as evidence of causation, even when they usually only identify a temporal association or indicate a chain of risk. The fact that low levels of alcohol consumption are associated with reduced dementia risk may be interpreted to indicate that alcohol has a 'protective' effect against dementia. However, it is also possible that abstainers, due to selection effects, have an increased risk of dementia, rather than moderate drinkers having a reduced risk. If this were the case, it would suggest that alcohol itself is not affecting cognition, but that drinking behavior is associated with other subject characteristics that influence cognitive decline. Without knowledge of the true mechanisms by which alcohol affects brain function and the selection effects pertaining to the different groups, causal interpretations must be made with caution.

Alcohol Exposure and Cognitive

Development

\section{Short-Term Effects versus Long-Term Change}

Risk factors for cognitive decline and dementia may have different or even opposite short-term and long-term effects. In terms of the taxonomy provided by $\mathrm{Li}$ et al. [47], the effects of risk factors may be viewed according to the manner in which they influence day-to-day fluctuations in performance, versus leading to long-term variation in the structuring of the organism. Laboratory studies of alcohol consumption in relation to cognitive test performance have shown that moderate levels of alcohol consumption negatively affect executive type functions [48], whereas the longer term follow-up studies suggest that low levels of consumption may have more general health benefits [48]. It therefore appears that the short-term decrements from exposure to alcohol in laboratory studies may be outweighed by the long-term changes caused by chronic exposure. This conundrum indicates that the time scale used to examine the association between a risk factor and an outcome may result in different conclusions being drawn about its effects. The short-term behavioral effects must be distinguished from long-term biological changes in structure and function. Environmental exposures may lead to positive or negative adaptation of the organism, with different degrees of reversibility on different time scales.

\section{Individual Differences: Comorbidity, Genetics and Sex Differences}

Another consideration for interpreting the influence of risk factors on level or rate of change in cognitive performance or on risk of dementia is the influence of other individual differences that may confound or moderate results. In particular, comorbid physical and mental health conditions [49], sex differences, and genetic factors have all been shown to moderate risk factors and cognitive outcomes.

Comorbid medical conditions may modify the influence of risk factors by directly affecting the level of the risk factor, by affecting the cognitive outcome, or through a combination of the two. For example, some diseases lead to a lowering of cholesterol. This means that individuals with initially high cholesterol may end up with lower levels due to diseases that also increase the risk of dementia [49]. Alcohol use is closely linked with other aspects of physical and particularly mental ill-health [50], indicating the need to study patterns of comorbidity among disorders when attempting to isolate the effect of alcohol consumption on cognitive function or cognitive decline.

Gerontology 2008;54:283-291 
Treatments for comorbid physical and mental health problems may also moderate the effect of a risk factor on cognitive function. Older adults frequently use both alcohol and prescription medications. Recent publications show that $86.9 \%$ of Finnish adults aged 75 years or over used both alcohol and medications on a regular basis including those known to have an interaction with alcohol [51], and data from an American prescription medicine assistance program for older persons found that $19 \%$ were using alcohol-interactive medications concomitantly with alcohol [52]. Alcohol may interact with medications to reduce cognitive performance.

The comorbid effects of genetic factors may moderate or interact with risk factors to alter their potential level of risk. An example of this is seen in the Cardiovascular Health Study in relation to stroke risk [53, 54]. This study showed that among men without the APOE*E4 genotype, drinking up to two drinks per day did not increase risk of stroke. However, in men with the APOE*E4 genotype, even moderate levels of drinking (1-2 drinks per day) were associated with increased risk of stroke. Another study that followed Finnish adults from mid-life for 23 years found that risk of dementia was increased in those with the $\mathrm{APOE}^{*} \mathrm{E}$ allele in a dose-response relationship, whereas an inverse association was found between alcohol consumption and risk of developing mild cognitive impairment in those not carrying the $\mathrm{APOE}^{*} \mathrm{E}$ allele [55].

Specific risk factors for cognitive decline may affect males and females differently. In general, it appears that alcohol is more toxic for women than for men, and this is recognized in the different criteria for classifying men and women into categories of mild, moderate, heavy, hazardous, and harmful drinkers [56]. There is a lack of epidemiological data on sex differences in the effects of alcohol on the brain, although results from case-control studies indicate greater negative effects of alcohol on the female brain $[57,58]$. Possible explanations for this are that women achieve higher blood concentrations of alcohol due to smaller body size and lower concentrations of alcohol dehydrogenase in the gut, and hence reach a threshold for brain damage at lower levels of consumption than men [59].

Sex differences are also observed in cognitive abilities across the lifespan, even after adjusting for health habits [60]. Although effect sizes are small, women appear to have better episodic memory and men tend to perform better on spatial tasks [61]. This means that sex differences and interactions need to be fully evaluated when investigating potential influences on cognitive development.

\section{Contextual Factors Such as Cohort, Ethnicity, Diet, Climate, Environment, and Culture}

Cohort effects may influence the likelihood and nature of exposure. Historical circumstances relating to the regulation of neurotoxins, nutrition and educational opportunities may all differ between cohorts. In the case of alcohol, there is some evidence that consumption declines in later life, independent of cohort effects. Two American longitudinal studies have investigated the apparent decrease in alcohol consumption with age. A 19year follow-up of 14,127 adults aged 25-74 found that the decline of heavy drinking with age was not associated with cohort effects [62]. A 10-year follow-up of 1,291 adults aged 55-65 reported that medical conditions, physical symptoms, medication use and acute health events were associated with lower frequency and quantity of alcohol intake and a higher likelihood of abstinence [63]. These authors suggested that older adults with more health problems tend to curtail their alcohol consumption so that age differences are explained by physical health. There are well-established cohort effects in cognitive function, showing a general increase in cognitive performance [64]. Hence, the development of lifespan models of the relationship between a risk factor and cognitive outcome needs to incorporate the possibility of cohort effects on the level of cognitive function and person-environment interactions when drawing data from samples born in different eras.

Historical, social and ethnic environments influence the level of specific risk factors and may moderate their effect. For example, the type of alcohol consumed differs between countries and between regions within countries. Alcohol consumption also occurs in a social context and hence drinking behavior is highly correlated with other factors that occur in the social environment. Studies have shown that young nondrinkers appear to be more withdrawn and less sociable $[65,66]$ than drinkers. On the other hand, binge drinking is more common amongst males than females, and in adolescents and young adults compared to middle and later life [67].

\section{Cognitive Ability as a Determinant of Context, Exposure to Other Risk Factors, and Moderator of Chronic Disease}

An important tenet of the contextualized, dynamic lifespan approach is that cognitive ability may also be an important risk factor for later health outcomes [16]. Cog- 
nition may therefore be part of a chain of risk factors that influence cognitive decline. That is, cycles or chains of risk factors for cognitive decline are moderated by education and cognitive ability at various stages of the life course. Rather than viewing relationships among risk factors for cognitive development as simple cause and effect, the dynamic approach to lifespan development incorporates self-determining cycles that interact with environmental, genetic, social and historical factors.

Cognitive ability is associated with better social environments and neighborhoods and higher levels of education in children which thus influence the intergenerational cycle [68]. Higher cognitive ability is likely to be associated with occupations that involve fewer exposures to toxins. It is also associated with a lower likelihood of adopting certain health behaviors that are risk factors for dementia, such as smoking. It is possible that patterns of alcohol consumption (and hence alcohol exposure) are associated with the level of cognitive ability prior to any effect of alcohol on the brain function or cognitive performance [69].

\section{Conclusion}

The association between alcohol and cognitive development has been used to illustrate the need for highly specific content knowledge for developing comprehen- sive models of cognitive aging within a contextualized, dynamic approach to lifespan epidemiology. Understanding the cognitive and behavioral factors that moderate exposures enables the identification of the different levels at which interventions may occur to reduce exposures or increase cognitively enhancing environments or behaviors. The same exposure (e.g. alcohol) may affect brain and cognition from either direct environmental influence (e.g. FAS) or via behavior of the individual (alcohol consumption). Prevention of exposure therefore requires (a) identification of the individual characteristics and ecological or contextual factors that give rise to the exposure, and (b) identification of strategies for changing exposures by changing the environment or behavior.

This review has proposed a contextualized, dynamic life course approach as a means of integrating the range of theoretical and methodological factors that are specific to the study of risk factors for cognitive growth and decline. This approach views cognitive development over the life course as emerging from a social and historical context that interacts with individual biology and behavior.

\section{Acknowledgements}

I thank Lesley Ross and Richard Burns for comments on the manuscript. Kaarin Anstey is funded by a National Health and Medical Research Council Fellowship, No. 366756.

\section{References}

1 Blane D, Netuveli G, Stone J: The development of life course epidemiology. Rev Epidemiol Sante Publique 2007;55:31-38.

-2 Kaplan GA, Baltrus PT, Raghunathan TE: The shape of health to come: prospective study of the determinants of 30-year health trajectories in the Alameda County Study. Int J Epidemiol 2007;36:542-548.

-3 Ramsay SE, Whincup PH, Morris RW, Lennon LT, Wannamethee SG: Are childhood socio-economic circumstances related to coronary heart disease risk? Findings from a population-based study of older men. Int J Epidemiol 2007;36:560-566.

4 Batty GD, Alves JG, Correia J, Lawlor DA: Examining life-course influences on chronic disease: the importance of birth cohort studies from low- and middle- income countries. An overview. Braz J Med Biol Res 2007;40: 1277-1286.

5 Kuh D, Ben-Shlomo Y, Lynch J, Hallqvist J, Power C: Life course epidemiology. J Epidemiol Community Health 2003;57:778-783.
-6 Nicolau B, Thomson WM, Steele JG, Allison PJ: Life-course epidemiology: concepts and theoretical models and its relevance to chronic oral conditions. Community Dent Oral Epidemiol 2007;35:241-249.

-7 Schaie KW: A general model for the study of development problems. Psychol Bull 1965; 64:92-107.

8 Horn JL: The aging of human abilities; in Wolman BB (ed): Handbook of Developmental Psychology. Englewood Cliffs, Prentice Hall, 1982, pp 847-869.

-9 Baltes PB, Reinert G: Cohort effects in cognitive development of children as revealed by cross-sectional sequences. Dev Psychol 1969; 1:169-177.

10 Baltes PB, Baltes MM, Reinert G: Relationship between time of measurement and age in cognitive development of children - application of cross-sectional sequences. Hum Dev 1970;13:258.
11 Baltes PB, Staudinger UM, Lindenberger U: Lifespan psychology: theory and application to intellectual functioning. Annu Rev Psychol 1999;50:471-507.

12 Whalley LJ, Deary IJ, Starr JM, Wahle KW, Rance KA, Bourne VJ, Fox HC: n-3 fatty acid erythrocyte membrane content, APOE epsilon 4 , and cognitive variation: an observational follow-up study in late adulthood. Am J Clin Nutr 2008;87:449-454.

13 Whalley LJ, Deary IJ, Appleton CL, Starr JM: Cognitive reserve and the neurobiology of cognitive aging. Aging Res Rev 2004;3:369382.

14 Horn JL: Remodelling old models of intelligence; in Wolman B (ed): Handbook of Intelligence: Theories, Measurements and Applications. New York, Wiley, 1985.

15 Hensch TK: Controlling the critical period. Neurosci Res 2003;47:17-22.

16 Deary IJ, Der G: Reaction time explains IQ's association with death. Psychol Sci 2005;16: 64-69. 
17 Card NA, Little TD, Bovaird JA: Modeling ecological and contextual effects in longitudinal studies of human development; in Modeling Contextual Effects in Longitudinal Studies. Mahwah, Lawrence Erlbaum Associates, 2007, pp 1-11.

18 Widaman K: Intrauterine environment affects infant and child outcomes; in Little TD, Bovaird JA, Card NA (eds): Modeling Contextual Effects in Longitudinal Studies. Mahwah, Lawrence Erlbaum, 2007, pp 387436.

19 Chen WJ, Maier SE, Parnell SE, West JR: Alcohol and the developing brain: neuroanatomical studies. Alcohol Res Health 2003;27: 174-180.

20 Wacha VH, Obrzut JE: Effects of fetal alcohol syndrome on neuropsychological function. J Dev Phys Disabil 2007;19:217-226.

21 West JR, Chen WJ, Pantazis NJ: Fetal alcohol syndrome: the vulnerability of the developing brain and possible mechanisms of damage. Metab Brain Dis 1994;9:291-322.

22 Barker DJ, Osmond C: Infant mortality, childhood nutrition, and ischaemic heart disease in England and Wales. Lancet 1986; 1:1077-1081.

23 Whalley LJ, Dick FD, McNeill G: A lifecourse approach to the aetiology of late-onset dementias. Lancet Neurol 2006;5:87-96.

24 Del Boca FK, Darkes J: The validity of selfreports of alcohol consumption: state of the science and challenges for research. Addiction 2003;98(suppl 2):1-12.

25 Dawson DA: Methodological issues in measuring alcohol use. Alcohol Res Health 2003; 27:18-29.

-26 Schmidt L, Room R: Cross-cultural applicability in international classifications and research on alcohol dependence. J Stud Alcohol 1999;60:448-462.

-27 Stampfer MJ, Kang JH, Chen J, Cherry R, Grodstein F: Effects of moderate alcohol consumption on cognitive function in women. New Engl J Med 2005;352:245-253.

28 Deng J, Zhou DH, Li J, Wang YJ, Gao C, Chen M: A 2-year follow-up study of alcohol consumption and risk of dementia. Clin Neurol Neurosurg 2006;108:378-383.

-29 Dassanayake T, Weerasinghe V, Dangahadeniya U, Kularatne K, Dawson A, Karalliedde L, Senanayake N: Cognitive processing of visual stimuli in patients with organophosphate insecticide poisoning. Neurology 2007;68:2027-2030.

-30 Hofer SM, Sliwinski MJ: Understanding ageing. An evaluation of research designs for assessing the interdependence of ageing-related changes. Gerontology 2001;47:341-352.

- 31 Rodgers B, Windsor TD, Anstey KJ, Dear KB, F Jorm A, Christensen H: Non-linear relationships between cognitive function and alcohol consumption in young, middle-aged and older adults: the PATH Through Life Project. Addiction 2005;100:1280-1290.
32 Wright CB, Elkind MS, Luo X, Paik MC, Sacco RL: Reported alcohol consumption and cognitive decline: the northern Manhattan study. Neuroepidemiology 2006;27:201207.

33 Naissides M, Mamo JC, James AP, Pal S: The effect of chronic consumption of red wine on cardiovascular disease risk factors in postmenopausal women. Atherosclerosis 2006; 185:438-445.

-34 Anstey KJ, Windsor TD, Rodgers B, Jorm $\mathrm{AF}$, Christensen $\mathrm{H}$ : Lower cognitive test scores observed in alcohol abstainers are associated with demographic, personality, and biological factors: the PATH Through Life Project. Addiction 2005;100:1291-1301.

35 Espeland MA, Gu L, Masaki KH, Langer RD, Coker LH, Stefanick ML, Ockene J, Rapp SR: Association between reported alcohol intake and cognition: results from the Women's Health Initiative Memory Study. Am J Epidemiol 2005; 161:228-238.

- 36 Mukamal KJ, Longstreth WT, Mittleman MA, Crum RM, Siscovick DS: Alcohol consumption and subclinical findings on magnetic resonance imaging of the brain in older adults: the cardiovascular health study. Stroke 2001;32:1939-1946.

37 den Heijer T, Vermeer SE, van Dijk EJ, Prins ND, Koudstaal PJ, van Duijn CM, Hofman A, Breteler MM: Alcohol intake in relation to brain magnetic resonance imaging findings in older persons without dementia. Am J Clin Nutr 2004;80:992-997.

38 Ding JZ, Nieto FJ, Beauchamp NJ, Longstreth WT, Manolio TA, Hetmanski JB, Fried LP: A prospective analysis of risk factors for white matter disease in the brain stem. Neuroepidemiology 2003;22:275-282.

39 Britton A, Singh-Manoux A, Marmot M: Alcohol consumption and cognitive function in the Whitehall II Study. Am J Epidemiol 2004;160:240-247.

40 Bond GE, Burr R, McCurry SM, Graves AB Larson EB: Alcohol, aging, and cognitive performance in a cohort of Japanese Americans aged 65 and older: the Kame Project. Int Psychogeriatr 2001;13:207-223.

-41 Galanis DJ, Joseph C, Masaki KH, Petrovitch $\mathrm{H}$, Ross GW, White L: A longitudinal study of drinking and cognitive performance in elderly Japanese American men: the Honolulu-Asia Aging Study. Am J Public Health 2000;90:1254-1259.

-42 Elias PK, Elias MF, D’Agostino RB, Silbershatz $\mathrm{H}$, Wolf PA: Alcohol consumption and cognitive performance in the Framingham Heart Study. Am J Epidemiol 1999;150:580 589.

43 Bates ME, Tracy JI: Cognitive functioning in young 'social drinkers': is there impairment to detect? J Abnorm Psychol 1990;99:242249.
44 Ganguli M, Vander Bilt J, Saxton JA, Shen C, Dodge $\mathrm{HH}$ : Alcohol consumption and cognitive function in late life: a longitudinal community study. Neurology 2005;65:12101217.

45 Bond GE, Burr RL, McCurry SM, Rice MM, Borenstein AR, Larson EB: Alcohol and cognitive performance: a longitudinal study of older Japanese Americans. The Kame Project. Int Psychogeriatr 2005;17:653-668.

46 Mukamal KJ, Massaro JM, Ault KA, Mittleman MA, Sutherland PA, Lipinska I, Levy D, D’Agostino RB, Tofler GH: Alcohol consumption and platelet activation and aggregation among women and men: the Framingham Offspring Study. Alcohol Clin Exp Res 2005;29:1906-1912.

47 Li SC, Huxhold O, Schmiedek F: Aging and attenuated processing robustness. Evidence from cognitive and sensorimotor functioning. Gerontology 2004;50:28-34.

48 Weissenborn R, Duka T: Acute alcohol effects on cognitive function in social drinkers: their relationship to drinking habits. Psychopharmacology 2003;165:306-312.

49 Launer LJ: The epidemiologic study of dementia: a life-long quest? Neurobiol Aging 2005;26:335-340.

50 Andrews G, Henderson S, Hall W: Prevalence, comorbidity, disability and service utilisation: overview of the Australian National Mental Health Survey. Br J Psychiatry 2001;178:145-153.

-51 Aira M, Hartikainen S, Sulkava R: Community prevalence of alcohol use and concomitant use of medication - a source of possible risk in the elderly aged 75 and older? Int J Geriatr Psychiatry 2005;20:680-685.

52 Pringle KE, Ahern FM, Heller DA, Gold CH, Brown TV: Potential for alcohol and prescription drug interactions in older people. J Am Geriatr Soc 2005;53:1930-1936.

53 Mukamal KJ, Ascherio A, Mittleman MA, Conigrave KM, Camargo CA Jr, Kawachi I, Stampfer MJ, Willett WC, Rimm EB: Alcohol and risk for ischemic stroke in men: the role of drinking patterns and usual beverage. Ann Intern Med 2005;142:11-19.

54 Mukamal KJ, Chung H, Jenny NS, Kuller LH, Longstreth WT Jr, Mittleman MA, Burke GL, Cushman M, Beauchamp NJ Jr, Siscovick DS: Alcohol use and risk of ischemic stroke among older adults: the cardiovascular health study. Stroke 2005;36:18301834.

55 Anttila T, Helkala EL, Viitanen M, Kareholt I, Fratiglioni L, Winblad B, Soininen H, Tuomilehto J, Nissinen A, Kivipelto M: Alcohol drinking in middle age and subsequent risk of mild cognitive impairment and dementia in old age: a prospective population based study. BMJ 2004;329:539.

56 National Health and Medical Research Council: Australian Alcohol Guidelines: Health Risks and Benefits. Canberra, NHMRC, 2001. 
-57 Pfefferbaum A, Rosenbloom M, Deshmukh A, Sullivan EV: Sex differences in the effects of alcohol on brain structure. Am J Psychiatry 2001;158:188-197.

58 Anstey KJ, Jorm AF, Reglade-Meslin C, Maller J, Kumar R, von Sanden C, Windsor TD, Rodgers B, Wen W, Sachdev P: Weekly alcohol consumption, brain atrophy, and white matter hyperintensities in a community-based sample aged 60 to 64 years. Psychosom Med 2006;68:778-785.

-59 Hommer DW, Momenan R, Kaiser E, Rawlings RR: Evidence for a gender-related effect of alcoholism on brain volumes. Am J Psychiatry 2001;158:198-204.

-60 Jorm AF, Anstey KJ, Christensen H, Rodgers $\mathrm{B}$ : Gender differences in cognitive abilities: the mediating role of health state and health habits. Intelligence 2004;32:7-23.

61 de Frias C, Nilsson LG, Herlitz A: Sex differences in cognition are stable over a 10 -year period in adulthood and old age. Aging Neuropsychol Cogn 2006;13:574-587.
62 Karlamangla A, Zhou K, Reuben D, Greendale G, Moore A: Longitudinal trajectories of heavy drinking in adults in the United States of America. Addiction 2006;101:9199.

63 Moos RH, Brennan PL, Schutte KK, Moos BS: Older adults' health and changes in latelife drinking patterns. Aging Ment Health 2005;9:49-59.

64 Verhaeghen P: Aging and vocabulary scores: a meta-analysis. Psychol Aging 2003;18:332339.

65 Leifman H, Kuhlhorn E, Allebeck P, Andreasson S, Romelsjo A: Abstinence in late adolescence - antecedents to and covariates of a sober lifestyle and its consequences. Soc Sci Med 1995;41:113-121.
66 Rodgers B, Korten AE, Jorm AF, Christensen $\mathrm{H}$, Henderson S, Jacomb PA: Risk factors for depression and anxiety in abstainers, moderate drinkers and heavy drinkers. Addiction 2000;95:1833-1845.

67 Kuntsche EN: Progression of a general substance use pattern among adolescents in Switzerland? Investigating the relationship between alcohol, tobacco, and cannabis use over a 12-year period. Eur Addict Res 2004; 10:118-125

68 O'Brien Caughy M: Health and environmental effects on the academic readiness of school-age children. Dev Psychol 1996;32: 515-522.

69 Taylor MD, Hart CL, Davey Smith G, Starr JM, Hole DJ, Whalley LJ, Wilson V, Deary IJ: Childhood mental ability and smoking cessation in adulthood: prospective observational study linking the Scottish Mental Survey 1932 and the Midspan studies. J Epidemiol Community Health 2003;57:464465 . 